\title{
Diabetic Retinopathy Detection
}

\author{
V. Sudha, K. Priyanka, T. Suvathi Kannathal, S. Monisha
}

\begin{abstract}
Diabetic retinopathy is becoming a more prevalent disease in diabetic patients nowadays. The surprising fact about the disease is it leaves no symptoms at the beginning stage and the patient can realize the disease only when his vision starts to fall. If the disease is not found at the earliest it leads to a stage where the probability of curing the disease is less. But if we find the disease at that stage, the patient might be in a situation of losing the vision completely. Hence, this paper aims at finding the disease at the earliest possible stage by extracting two features from the retinal image namely Microaneurysms which is found to be the starting symptom showing feature and Hemorrhage which shows symptoms of the other stages. Based on these two features we classify the stage of the disease as normal, beginning, mild and severe using convolutional neural network, a deep learning technique which reduces the burden of manual feature extraction and gives higher accuracy. We also locate the position of these features in the disease affected retinal images to help the doctors offer better medical treatment.
\end{abstract}

Keywords: Microneurysm; diagnose at the earliest stage; Hemorrhage; locate features; convolutional neural networks.

\section{INTRODUCTION}

Image Processing in computer science is very rapidly growing area of application which is used to process the digital images and manipulate through algorithms. It is also used to enhance and extract useful information from the images which undergone the process based on characteristics and features associated in that image. Also, Image processing plays an important role in our daily life and in the field of science which includes remote sensing, computer vision, face detection, finger print detection, feature extraction, biometric verification, signature recognition, biomedical image enhancement and medical palmistry. Image is the collection of pixels each holding a single value. The values held by the pixels are the intensity of light falling on that position in the image. The methodology in Image processing follows (1) Image acquisition, (2) preprocessing (3) edge-detection, (4) segmentation, (5) Image restoration and (6) output processed image.

Revised Manuscript Received on April 11, 2020.

* Correspondence Author

V. Sudha*, Assistant professor, Computer science and engineering Kumaraguru College of technology

Coimbatore, India, Email at: sudha.v.cse@kct.ac.in

K. Priyanka, Computer science and engineering Kumaraguru College of technology Coimbatore, India,Email at: priyanka.16cs@kct.ac.in

T. Suvathi Kannathal, Computer science and engineering Kumaraguru College of technology Coimbatore, India, Email at: suvathi.16cs@kct.ac.in

S. Monisha, Computer science and engineering Kumaraguru College of technology Coimbatore, India, Email at: monisha.16cs@kct.ac.in

(C) The Authors. Published by Blue Eyes Intelligence Engineering and Sciences Publication (BEIESP). This is an open access article under the CC BY-NC-ND license (http://creativecommons.org/licenses/by-nc-nd/4.0/)
The sequence of image processing has an approach of storing the digital image on the system by sampling the image at regular grid. The color and intensity of each pixel is converted into numeric values and stored in the computer. The process of digital image processing is faster and costeffective. No more fixing of chemical processing are needed to process images. To improve the quality of image and visual effect of people is the main purpose of image processing. It takes the low-quality input image, trains, classifies, and tests with various algorithms to display the image with high quality. In each image the accuracy level is indicated according to the processing aspects of each algorithm. This is an optimized workflow to reduce the time during the operations of image processing. In this paper, image processing in one of the medical field problems, is explained.

Medical image processing creates the visual representations of the interior body for clinical analysis and classifies them based on various mathematical formulations. In visual perception the elements include human eye, retinal surface, sampling, spatial, resolution, grey levels, labelling etc. Also, it reduces the noise, segment and enhances them for improved quality which accurately detects the focus of diseases and communicates medical and pathological information of particular image by visual representation. In this paper we are going to discuss about the disease caused due to diabetics called Diabetic

Retinopathy. Also, includes how image processing techniques helps to detect the disease by taking the retinal fundus images of diabetic affected people.

Diabetic Retinopathy (DR) is a retinal bruise, caused by elevation of blood sugar levels, which leads to vision impairment. According to an estimation the number of people with blindness due to diabetic retinopathy is 400,000 (0-1.5 million), with low vision is 2.6 million since 1990 . Diabetic Retinopathy is a condition which causes damages in retina due to type 1 diabetics.

According to the estimation of The World Health Organization (WHO) diabetics affected people are around $8.4 \%$. About $90 \%$ of people are not aware of fundamental evaluation for Diabetic Retinopathy. Mostly people between ages 20 to 64 are affected to this blindness.

The blood supply to the retina by vessels is susceptible to unrestrained blood sugar level. It also leads to crumbling and blockage of vessels due to insufficient supply of oxygen and cause severe injury. Microaneurysms are the earliest sign of Diabetic Retinopathy. There are two stages namely proliferative Diabetic Retinopathy (PDR) and nonproliferative Diabetic Retinopathy (NPDR). At the stage of NDPR the retinal blood vessels gets damaged and becomes wet and swollen. PDR stage occurs when abnormal blood vessels appear in various areas in retina. Detection at the early stage of this disease is very complex but essential.

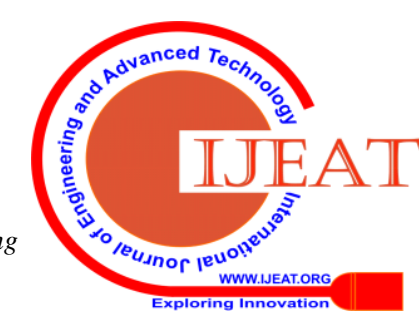


It's very important for regular screening of Diabetic Retinopathy to prevent further complication. Many researches from different medical industry are undergone for this purpose. It could be detected by machine learning and image processing. The retina of the diabetic affected patients is captured using fundus photography tool. It is also useful for monitoring the improvement or progression of diabetic retinopathy. Several image processing techniques includes filter, segmentation, classification and image enhancement has been developed for the early detection of Diabetic Retinopathy by the features of exudates, blood vessels, hemorrhages and Microaneurysms. These techniques are very useful to extract the features by sharpening, blurring, reducing noise etc. Convolutional Neural Network plays a major role for this disease detection. These techniques are explained elaborately below in this paper.

\section{LITERATURE SURVEY}

Nandana Prabhu et al., [1] have proposed a system for Diabetic Retinopathy detection based on the presence of the feature that shows the symptoms of the disease. The system makes use of fundus images, the bright lesions on the retina and the exudates are extracted as they indicate the symptoms of the disease. Based on the features extracted various stages of the disease is detected using hierarchical classification

.They have emphasized the needs of detection system due to increased number of cases and less ophthalmologists to treat, and the system has resulted in high accuracy in sensitivity and specificity.

Anupriyaa Mukherjee et al., [2] have discussed about various image processing techniques to classify the normal and diseased image in order to find the problems in the detection system. Preprocessing and detection of the various features such as Optic disc detection, Blood vessels extraction, and Exudates detection are applied. They have proposed a low cost system to diagnose at an early stage with a high accuracy rate; the algorithm could be used even for poor computing systems.

Imran Qureshi et al., [3] have presented about review of CAD systems in diagnosing Diabetic Retinopathy. They have also discussed about all the CAD systems which have been developed for various needs such computation Intelligence and Image processing techniques. They also conducted a survey on screening algorithms various research papers in detection and their challenges and results. Demonstration the challenges and automated DR methodologies along with possible solutions were demonstrated.

Yogesh Kumaran, Chandrashekar M. Patil [4] have presented a brief survey in detecting DR using different preprocessing and segmentation techniques as it is difficult to process the raw fundus images by machine learning algorithms. They have given brief view from the nutshell in order to facilitate others on recent advancement and research for their work. This can also help in the insight detection which is based on the work of researches in the field.

Nisha A. Panchal et al., [5] have surveyed various techniques involved in detection of Diabetic Retinopathy. Various classifiers are used to classify the features such as blood vessels, optic disc; exudates (EXs), hemorrhages
(HMs).The survey papers used have various standalone methods to detect using fundus images. They have also given accuracy in terms of specificity and sensitivity excluding the processing time.

Ankita Gupta, Rita Chhikara [6] have compared the various machine learning algorithms experimental results. The parameters focused are like sensitivity, specificity, Area under Curve (AUC), Accuracy. The review detects DR approaching Blood vessels segmentation and Identification of lesions. The obtained results were also compared with deep neural network. Out of the various analyses best technique is provided. Moreover has provided high efficiency in detection of the desired features.

M.Kamaladevi et al., [7] have proposed an automatic detection framework for Diabetic Retinopathy. They have extracted the features from the affected retinal images and detected them. Methodologies such as feature extraction is carried out and classified using classifiers such as Adaboost, Gradientboost, RandomForest, Voting classifier in the system. Over them prediction has remained predominated in RandomForest with a higher rate of accuracy.

Salman Sayed et al., [8] have compared the detection of Diabetic Retinopathy in the fundus images using the models Probabilistic Neural Network (PNN) and Support vector machines (SVM).Initially through the preprocessing followed by machine learning techniques. Preprocessing techniques

such as Grayscale conversion, Adaptive Histogram Equalization, Discrete Wavelet Transform, Matched filter and Fuzzy C-means segmentation are used to treat the poor image quality. Further the yielded result of both the techniques are compared and analyzed. Detection accuracy of Support Vector Machine (SVM) out performed than Probabilistic Neural Network PNN).

Prabhjot Kaur et al., [9] have proposed a model to detect Diabetic retinopathy using the three major phases such as preprocessing, segmentation and classification. They have implemented the model in MATLAB and based on certain parameters results are analyzed. In this work NN classification approach is proposed for of the detection the proposed model and SVM classification is compared and analyzed. The result thus produced is much more efficient compared an optimized with the use of NN.

Kanika Verma et al., [10] have proposed a system to detect blood vessel, identify hemorrhages and classify different stages of diabetic retinopathy into normal, moderate and non- proliferative diabetic retinopathy (NPDR). The retinal vascular were segmented using density analysis and through bounding box techniques. Finally classified by using the area and perimeter of blood vessels and hemorrhages by Random Forests technique.

Manjusha Nair, Dhirendra Mishra [11] has presented the subjective and objective analysis methods. They have attempted to classify the various levels of severity of Diabetic Retinopathy. They have used clustering and thresholding methods. Spatial Domain and Frequency Domain are implemented for calculating Feature Vectors. Thresholding is finally used to classify and also produced an encouraging result in terms of Sensitivity, Specificity, and Accuracy.

Published By:

Blue Eyes Intelligence Engineering

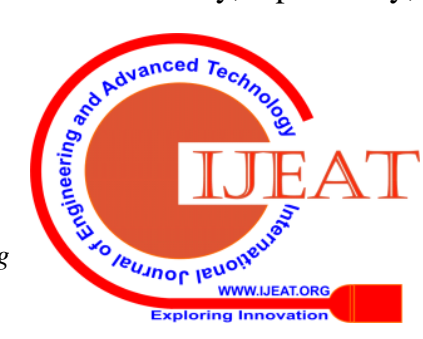


Muhammad Waseem Khan [12] has presented recent works for feature detection using image processing technique for diabetic retinopathy lesion and these are further evaluated. Also discussed about various image processing techniques and different lesion with appropriate result. Further hybrid method can be used in order to get higher accuracy. This can help and diagnose at an higher rate. Early detection and prevention are much more effective in treating them and is efficient. Carson Lam, M et al., [13] have demonstrated to recognize the different stages of diabetic retinopathy on a color fundus images using convolutional neural networks (CNNs). The network model also obtained a higher rate of validation sensitivity. They have also demonstrated primary errors that occurred and misclassified due to the inability of CNN. Preprocessing with adaptive histogram equalization and a dataset fidelity verification improved the subtle features recognition. From the above literature survey, we can see that most of the existing system detects and classifies the disease but it doesn't locate the features showing symptoms in the retinal images for better analyses. Also, the number of features taken into account for detecting the disease is more. Also, the early stage symptom showing feature is rarely extracted for detection.

\section{PROPOSED SYSTEM}

Here, we are introducing a novel approach for detecting and classifying the diabetic retinopathy disease at the earliest stage by extracting the features such as Hemorrhages and Microaneurysms from the retinal images using Convolutional Neural Network method. So, we attempt to reduce the number of features to two including Microaneurysms which is the earliest symptom of the disease. We exclude the blood vessel feature since it shows symptoms only after Microaneurysms. The main motive of our project is to detect the disease at a stage where we still have possibilities to cure the disease and to locate the symptom showing feature in the retinal images. Since, the patients realizes no symptoms until their vision turns to a little blinder and have only minimum chance of getting cured, the best way to detect the disease is as early as possible which has been covered in this paper.

\section{A. Our method of approach - Brief introduction}

The first step is to input the images to the convolutional neural network (refer Fig.1). But we are not training the network straightaway with the input images. We need to do some preprocessing before training the model. The need for preprocessing is to improve the image data for enhancing the features of the image and to filter the random noises for better processing. Then we resize all the images downscale to level up images of different dimensions to a single dimension. After preprocessing phase is completed, the next step is to go for feature selection and feature extraction. Feature selection involves selecting a subset of features from the total image features in a way that this subset is itself enough to classify the images as one with Hemorrhage-Microaneurysms and as one without it which is normal. Extraction of features involves reducing the dimension of the selected subset of features. This is to reduce the size of input that is going to be fed for training the model. Feature extraction attempts to eliminate the redundant pixels or information from the selected subset of features so as to make it more suitable for further processing.

\section{B. Traditional classification algorithms versus Convolutional neural network}

In traditional methods, the process of training and testing flow starts from input of the image, then manual feature extraction followed by training and testing the model for detection and classification. But the advantage of convolutional neural network is that it does the feature selection and feature extraction by itself from end-to-end. So, there is no need to manually extract the features; instead input the images containing only those features and the rest is left up to the Convolutional Neural Network.

The Preprocessed image is directly fed into the Convolutional Neural Network for the reason stated above. And it does all the feature selection and extraction by subsampling the parts of the image through each layer of convolution and pooling.

Actually there are many numbers of convolution and pooling layer for better classification.

Finally, after many passes into these two layers it reaches the fully connected layer, the last layer of Convolutional Neural Network, where it builds the filters for classification of the image to normal and abnormal. Here stops the training part. The testing part also follows the same procedure but one more additional step it does is it performs an operation with the output of testing image values obtained from the fully connected layer with the values of filters which was constructed previously in the training part. If a match with higher score is found, then it's classified accordingly. Then from the abnormal images, hemorrhages and Microaneurysms are segmented.

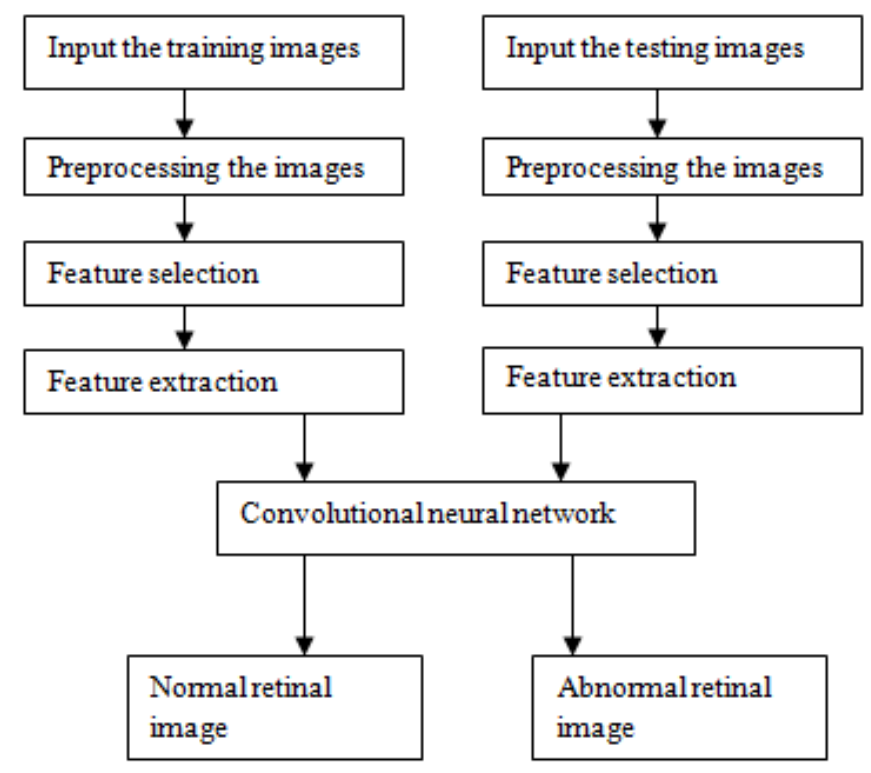

Fig.1 Block diagram of the work flow

\section{Preprocessing techniques}

We use two techniques of preprocessing in this paper. One is resizing or compression and the other is noise filtering.

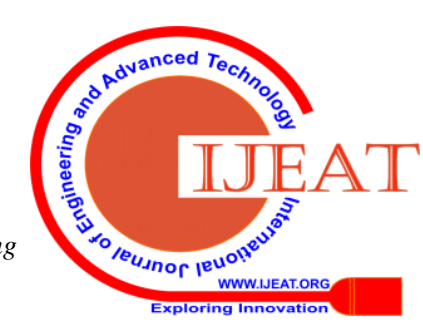




\section{Resizing the images}

The need for resizing is to convert all the varying dimensional (height and width) images to a single dimension. Because the training algorithm takes images which are of same height and width as input for better processing and thus reduces the processing time. Here in our dataset we have random dimensionality images such as $228 \times 221,998 \times 630$ and so on. Hence we resize the images to a height of 96 and width of 96 with depth as 3 that is three channels since we are using color images.

\section{Noise Filtering}

Noise is nothing but the random variation in color or brightness of the image. Here, the noise present in our image would be speckle noise which is a kind of noise that occurs due to improper illumination of light while capturing the medical images. So to remove the noise, median filter is used. This filter works by taking a window of size $3 \times 3$ and replaces the pixel value by the median of the values residing in the window. One advantage of median filter is that it preserves the edges while removing the noise.

\section{Feature selection, Feature extraction, Model training and testing using $\mathrm{CNN}$}

Before we pass our preprocessed image to the CNN, we need to convert the image to an array and map that array values in the range of 0 to 1 . We set the epoch as 235 to attain a deep network. The initial learning rate is kept as 1e3 which is the default value for Adam optimizer and the batch size is 32 . To train our model with more images since we have only 190 images for training, we generate more images from the existing dataset by passing parameters such as rotation range, width shift range, height shift range, shear range, zoom range and horizontal flip to the Image Data generator.

\section{1) Convolutional neural network}

CNN comes under deep learning where it simulates the actual functioning of the brain neurons which capture and recognize objects. The CNN consists of three layers namely the convolution layer, the pooling layer and the fully connected layer. We are using Smallervggnet architecture of CNN which performs really well on image recognition and classification. The vgg network uses 3x3 convolution window followed by max pooling and finally end up with fully connected layer prior to a softmax classifier.

\section{2) Convolution layer}

The convolution layer builds an image recognition classifier whose bias and weights are based on the concept of gradient descent. That means the activation map the layer builds should be able to classify the image properly at the same time it should be of less error. After constructing the activation map of size $3 \times 3$, it slides the window over the entire image matrix which is called convolving and replaces the image matrix with the new values obtained from convolving. The output is passed to the pooling layer.

\section{3) Pooling layer}

The pooling layer reduces the spatial volume of the output and we use Max pooling which means we take a pool of size $3 \times 3$ from the image matrix (output of 1st layer) and finds the highest number in the pool and replace the entire pool with that highest number. Thus by this way, we reduce the spatial volume. For first two passes we keep the pool size as $3 \times 3$ and gradually decreasing it to $2 \times 2$ in the next pass.

\section{4) Fully connected layer}

The fully connected input layer takes the output of the previous layers and flattens them to a single vector value. Then it applies weights to the features to classify the labels. At last it calculates probability for each of the labels. The label with high probability will be matched for the given input image. Thus by this way, we can get our detection and classification done.

\section{5) Dropout}

Dropout states how many nodes of the current layer need to be cut off or disconnected from the next layer. This is to ensure the redundancy exists naturally in the model so that no one specific node predicts the certain class of the image.

\section{6) Training the model}

On the whole, we first update the input shape of image as channel first. Then for Smallervggnet we pass parameters such as the convolution window size as $3 \times 3$, the number of filters it should built out of it as 32,for the next pass its 64 and for the next pass its 128 and the padding value is marked 'same' to preserve the dimension of the output as same as the input size. Next, the activation function used here ReLu, the Rectified Linear Unit, takes values which are either positive or zero. It also increases the non-linearity of the image. Then, we do batch normalization followed by max pooling of pool size 3x3. At last, the dropout is marked as 0.25 for convolution and pooling layer and it is 0.5 for fully connected layer. Dropout helps to control overfitting. Finally we end up with softmax classifier to return our predicted probabilities for each class label.

\section{7) Testing the model}

The same process starting from preprocessing till the last layer of CNN is followed again for the test images. Now an operation is performed with the output of the testing image values from the fully connected layer with the values of all filters that was constructed previously in the training part to calculate the probability. The highest probability value is taken as the correct match. Finally we segment the two features from the abnormal images.

\section{RESULT AND DISCUSSION}

We use python shell 3.6.8 for front end and tensor flow for CNN backend. To measure the accuracy of our training model we use some parameter as mentioned below,

\section{a) Binary cross entropy}

Binary cross entropy is a loss function which is used on problems involving binary answer like yes or no. Since we do multi-label classification, to find whether the example belongs to this class or not, we use binary cross entropy loss function.

\section{b) Overfitting and Underfitting}

Overfitting measures how my model performs so well such that it affects the result in a negative way. Overfitting occurs when our model learns the random noise and irrelevant details from the image dataset.

Underfitting measures how my model is performing less such that it could not predict the images correctly. Here we attempt to calculate the loss and accuracy of the model along with the loss and accuracy obtained from cross 
validating the model to check for underfitting and overfitting. In our project, after training the model, at the 235th epoch the learning rate is found to be 2s, training accuracy is 0.9375 , training loss is 0.2198 , value accuracy is 0.4773 and value loss is 0.6584 . From this we can infer that our training accuracy is greater than the value accuracy and the training loss is less than the value loss. So our trained model achieves low loss with limited overfitting with accuracy above $95 \%$ (95 to $100 \%$ ) in testing. So with a good training model we achieve better prediction accuracy for the testing images.

\section{CONCLUSION}

As we used Convolutional neural network methodology, every input image will be classified with the highest accuracy assuring no misclassification happens. We first trained the CNN to recognize lines, edges, corners etc. from the retinal image followed by recognizing small parts of a single feature which is then followed by recognizing a whole feature. By this way it learnt to identify Microaneurysms and hemorrhage features from the retinal image and thus classified the images accordingly to normal, beginning, mild and severe stages. Since we are extracting a feature which shows signs of the disease at the earliest stage, recovery chances will be high, thus vision of many people could be saved. After this classification, to help the doctors locate those features showing symptoms in the image, we automate marking those features in every classified abnormal retinal image. The proposed idea is not only helpful for Diabetic retinopathy affected people but can also be used by the melanoma and myeloid leukemia disease affected people. One more advantage of this system is, it can be implemented to achieve higher accuracy even with less number of dataset. We used only 20 to 50 images in each of the six classes and achieved accuracy above $95 \%$.

\section{REFERENCES}

1. Nandana Prabhu, Deepak Bhoir, Nita Shanbhag "Diabetic Retinopathy Screening using Machine Learning for Hierarchical Classification", International Journal of Innovative Technology and Exploring Engineering (IJITEE) Volume-8.

2. Anupriyaa Mukherjee, Diksha Rathore, Supriya Shree, Asst Prof. Shaik Jameel "Diagnosis of Diabetic Retinopathy", International. Journal of Engineering Research and Applications(IJERA) Volume5

3. Imran Qureshi, Jun Ma, and Qaisar Abbas "Recent Development on Detection Methods for the Diagnosis of Diabetic Retinopathy".

4. Yogesh Kumaran, Chandrashekar M. Patil "A Brief Review of the Detection of Diabetic Retinopathy in Human Eyes Using PreProcessing \& Segmentation Techniques", International Journal of Recent Technology and Engineering (IJRTE) Volume-7.

5. Nisha A. Panchal, Dr. Darshak G. Thakore, Dr. Tanmay D. Pawar "Detection of Diabetic Retinopathy: A Survey", International Journal for Research in Applied Science \& Engineering Technology (IJRASET) Volume-6.

6. Ankita Guptaa, Rita Chhikara “ Diabetic Retinopathy: Present and Past'

7. $\quad$ International Conference on Computational Intelligence and Data Science 2018 (ICCIDS)

8. M.Kamaladevi , S.SnehaRupa, T.Sowmya "Automatic Detection of Diabetic Retinopathy in Large Scale Retinal images”, International Journal of Pure and Applied Mathematics 2018 (IJPAM) Volume119.

9. Salman Sayed, Dr. Vandana Inamdar, Sangram Kapre "Detection of Diabetic Retinopathy using Image Processing and Machine Learning", International Journal of Innovative Research in Science, Engineering and Technology 2017.

10. Prabhjot Kaur, Somsirsa Chatterjee, Dilbag Singh "Neura Network Technique for Diabetic Retinopathy Detection", International Journal of Engineering and Advanced Technology (IJEAT) 2019 Volume-8.

11. Kanika Verma, Prakash Deep and A. G. Ramakrishnan "Detection

Retrieval Number: D7786049420/2020@BEIESP

and Classification of Diabetic Retinopathy using Retinal Images”, Journal gate Conference Paper December 2011.

12. Manjusha Nair, Dhirendra Mishra "Classification of Diabetic Retinopathy Severity Levels of Transformed images using Kmeans and Thresholding method", International Journal of Engineering and Advanced Technology 2019 (IJEAT)Volume-8.

13. Muhammad Waseem Khan "Diabetic Retinopathy Detection using Image Processing: ASurvey", International Journal Of Emerging Technology \& Research 2013 Volume-1.

14. Carson Lam, MD, Darvin Yi, Margaret Guo , Tony Lindsey" Automated Detection of Diabetic Retinopathy using Deep Learning".

\section{AUTHORS PROFILE}

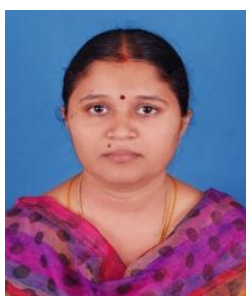

Mrs. V. Sudha received her BE degree in Computer Science and Engineering from VMKV Engineering College, Salem and ME degree in Computer Science and Engineering from CEG Campus, Anna University. She is currently pursuing her $\mathrm{PhD}$ in Anna University in the area of Theoretical Computer Science.

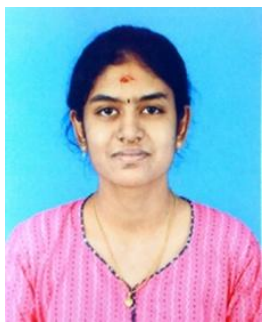

Ms. T. Suvathi Kannathal is doing her fina semester of undergraduation in computer science and engineering in Kumaraguru College of technology, Coimbatore. Having interest in app development she earned a credit completing zoho app development course and MongoDB; certified by NPTEL for the course 'operating systems' and undergone 'The Scrum fundamentals' training. Created team projects such as death registration app, music recognition system and designed an order placing website. Presented a game 'Hungerrandom' for the Artificial Intelligence techfest conducted in the campus which was selected among the top 5 teams and was reviewed by a member of IIT Mumbai. She secured 'Mahatma Gandhi merit scholarship' award three years consecutively for her academic excellence.

Ms. K. Priyanka is studying Bachelor of Engineering (final year) in computer science department. Her research works includes on her project namely Diabetic Retinopathy (DR) Participated in hackathon conducted by Forge Accelerator in Kct Tech Park, Coimbatore Developed a prototype namely classification of milk based on color, density and viscosity, which rewards an achievement.

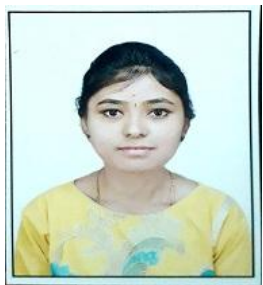

Ms. S. Monisha is studying Bachelor of engineering (final year) in computer science in Kumaraguru college of technology, Coimbatore. Participated in zoho app development training conducted in KCT. She worked on the research about the various existing Diabetic retinopathy detection system.
Published By:

Blue Eyes Intelligence Engineering

$\&$ Sciences Publication

(C) Copyright: All rights reserved. 